\title{
Energy and Momentum Distributions of Kantowski and Sachs Space-time
}

\author{
Ragab M. Gad ${ }^{1}$ and A. Fouad \\ Mathematics Department, Faculty of Science, \\ Minia University, 61915 El-Minia, EGYPT.
}

\begin{abstract}
We use the Einstein, Bergmann-Thomson, Landau-Lifshitz and Papapetrou energy-momentum complexes to calculate the energy and momentum distributions of Kantowski and Sachs space-time. We show that the Einstein and Bergmann-Thomson definitions furnish a consistent result for the energy distribution, but the definition of LandauLifshitz do not agree with them. We show that a signature switch should affect about everything including energy distribution in the case of Einstein and Papapetrou prescriptions but not in BergmannThomson and Landau-Lifshitz prescriptions.
\end{abstract}

\section{Introduction}

One of the most interesting and intricate problems still unsolved since the outset of general relativity is the energy-momentum localization. Einstein himself proposed the first energy-momentum complex in an attempt to define the local distribution of energy and momentum [1]. After this attempt, a plethora of different energy-momentum complexes were proposed, including formulations by Tolman [2], Papapetrou [3], Møller [4], Landau and Lifshitz [5], Weinberg [6], Bergmann-Thomson [7] and others. This approach was abandoned for a long time due to severe criticism for a number of reasons.

Recently, Virbhadra re-opened the subject of energy-momentum complexes [8]. He pointed out that though these complexes are non-tensors, they yield reasonable and consistent results for a given space-time. Aguirregabiria et al [9] found that for any metric of the Kerr-Schild class, several different definitions of the energy-momentum complex yield precisely the same results.Virbhadra [10] investigated whether or not these energy momentum complexes lead to the same results for the most general nonstatic spherically symmetric metric and found that they disagree. He noted that the energy-momentum complexes of Landau and Lifshitz, Papapetrou

\footnotetext{
${ }^{1}$ Email Address: ragab2gad@hotmail.com
} 
and Weinberg give the same results as in the Einstein definition if the calculations are performed in Kerr-Schild Cartesian coordinates. However, these energy-momentum complexes disagree if computations are done in "Schwarzschild Cartesian" coordinates". In a detail study of the question, Xulu [11] has confirmed this suggestion. He obtained the energy distribution for the most general non-static spherically symmetric using Møller's definition and found different results in general from those obtained using Einstein's definition. These results agree for the Schwarzschild, Vaidya and Janis-Newmann-Winicour space-times, but disagree for the ReissnerNordström space-time. Many authors had similarly successfully applied the aforementioned energy-momentum complexes to various black hole configurations [12].

It has been remained a controversial problem whether or not energy and momentum are localizable. There are different opinions on this subject. Contradicting the viewpoint of Misner et al. [13] that the energy is localizable only for spherical systems, Cooperstock and Sarracino [14] argued that if the energy localization is meaningful for spherical systems then it is meaningful for all systems. Bondi [15] expressed that a non-localizable form of energy is inadmissible in relativity and its location can in principle be found. These contradictory viewpoints bear significantly on the study of gravitational waves. It is an interesting question whether or not gravitational waves have energy and momentum content. In a series of papers, Cooperstock [16] hypothesized that in a curved space-time energy and momentum are confined to the region of non-vanishing energy-momentum tensor $T_{b}^{a}$ and consequently the gravitational waves are not carriers of energy and momentum in vacuum space-times. This hypothesis has neither been proved nor disproved. There are many results supporting this hypothesis (see for example, $[17,18]$ ).

In this paper we evaluate the energy and momentum distributions of the Kantowski and Sachs space-time, using Einstein, Bergmann-Thomson, Landau-Lifshitz and Papapetrou energy-momentum complexes.

Through this paper we use $G=1$ and $c=1$ units and follow the convention that Latin indices take value from 0 to 3 and Greek indices take value from 1 to 3 .

\footnotetext{
${ }^{2}$ Schwarzschild metric in "Schwarzschild Cartesian coordinates" is obtained by transforming this metric (in usual Schwarzschild coordinates $\{t, r, \theta, \phi\}$ ) to $\{t, x, y, z\}$ using $x=r \sin \theta \cos \phi, y=r \sin \theta \sin \phi, z=r \cos \theta$.
} 


\section{Kantowski and Sachs Space-time}

The standard representation of Kantowski and Sachs space-times are given by [19]

$$
d S^{2}=d t^{2}-A^{2}(t) d r^{2}-B^{2}(t)\left(d^{2} \theta+\sin ^{2} \theta d^{2} \phi\right),
$$

where the functions $A(t)$ and $B(t)$ are to be determined from the field equations.

The solutions of the Einstein field equations for the above metric were considered with dust source [19], but they were generalized (in fact earlier) to the general perfect fluid source [21].

From the geometrically point of view, this line element admits a four parameter continuous group of isometries which acts on space-like hypersurface, and has no three parameter subgroup that would be simply transitive on the orbits (for more detailed description see Kantowski and Sachs [19] and Collins [20]).

From the physical point of view, the metric (2.1) automatically defines an energy-momentum tensor of a fluid with anisotropic pressure, and the coordinates of (2.1) are comoving. The rotation and acceleration are zero, but if the source is to be a perfect fluid, then the shear is necessarily non-zero.

It is well known that if the calculations are performed in quasi-Cartesian coordinates, all the energy-momentum complexes give meaningful results. According to the following transformations

$$
r=\sqrt{x^{2}+y^{2}+z^{2}}, \quad \phi=\arctan \left(\frac{y}{x}\right),
$$

the line element (2.1) written in terms of quasi-Cartesian coordinates reads:

$$
d S^{2}=d t^{2}+\frac{B^{2}}{r^{2}}\left(d x^{2}+d y^{2}+d z^{2}\right)-\frac{1}{r^{2}}\left(A^{2}-\frac{B^{2}}{r^{2}}\right)(x d x+y d y+z d z)^{2} .
$$

For the above metric the determinant of the metric tensor and the con- 
travariant components of the tensor are given, respectively, as follows

$$
\begin{array}{cc}
\operatorname{det}(g) & =-\frac{A^{2} B^{4}}{r^{4}}, \\
g^{00} & =1, \\
g^{11} & =x^{2}\left(\frac{1}{B^{2}}-\frac{1}{r^{2} A^{2}}\right)-\frac{r^{2}}{B^{2}}, \\
g^{12} & =x y\left(\frac{1}{B^{2}}-\frac{1}{r^{2} A^{2}}\right), \\
g^{13} & =x z\left(\frac{1}{B^{2}}-\frac{1}{r^{2} A^{2}}\right), \\
g^{22} & =y^{2}\left(\frac{1}{B^{2}}-\frac{1}{r^{2} A^{2}}\right)-\frac{r^{2}}{B^{2}}, \\
g^{23} & =y z\left(\frac{1}{B^{2}}-\frac{1}{r^{2} A^{2}}\right), \\
g^{33} & =z^{2}\left(\frac{1}{B^{2}}-\frac{1}{r^{2} A^{2}}\right)-\frac{r^{2}}{B^{2}} .
\end{array}
$$

\section{Einstein's Energy-momentum Complex}

The energy-momentum complex as defined by Einstein [1] is given by

$$
\theta_{i}^{k}=\frac{1}{16 \pi} H_{i, l}^{k l}
$$

where the Einstein's superpotential $H_{i}^{k l}$ is of the form

$$
H_{i}^{k l}=-H_{i}^{l k}=\frac{g_{i n}}{\sqrt{-g}}\left[-g\left(g^{k n} g^{l m}-g^{l n} g^{k m}\right)\right]_{, m} .
$$

$\theta_{0}^{0}$ and $\theta_{\alpha}^{0}$ are the energy and momentum density components, respectively. The Einstein energy-momentum satisfies the local conservation law

$$
\frac{\partial \theta_{i}^{k}}{\partial x^{k}}=0
$$

The energy and momentum in the Einstein's prescription are given by

$$
P_{i}=\iiint \theta_{i}^{0} d x^{1} d x^{2} d x^{3} .
$$

Using the Gauss theorem we obtain

$$
P_{i}=\frac{1}{16 \pi} \iint H_{i}^{0 \alpha} n_{\alpha} d s
$$

where $n_{\alpha}=\left(\frac{x}{r}, \frac{y}{r}, \frac{z}{r}\right)$ are the components of a normal vector over an infinitesimal surface element $d s=r^{2} \sin \theta d \theta d \phi$. 
The required non zero components of $H_{i}^{k l}$ for the line element (2.1) are given by

$$
\begin{gathered}
H_{0}^{01}=\frac{2 x}{A r^{4}}\left(A^{2} r^{2}+B^{2}\right), \\
H_{0}^{02}=\frac{2 y}{A r^{4}}\left(A^{2} r^{2}+B^{2}\right), \\
H_{0}^{03}=\frac{2 z}{A r^{4}}\left(A^{2} r^{2}+B^{2}\right), \\
H_{1}^{01}=\frac{2 B}{r^{2}}\left(\frac{x^{2}}{r^{2}}(\dot{A} B-A \dot{B})-(\dot{A} B+A \dot{B})\right), \\
H_{2}^{01}=H_{1}^{02}=\frac{2 x y B}{r^{4}}(\dot{A} B-A \dot{B}), \\
H_{3}^{01}=H_{1}^{03}=\frac{2 x z B}{r^{4}}(\dot{A} B-A \dot{B}), \\
H_{2}^{02}=\frac{2 B}{r^{2}}\left(\frac{y^{2}}{r^{2}}(\dot{A} B-A \dot{B})-(\dot{A} B+A \dot{B})\right), \\
H_{3}^{02}=H_{2}^{03}=\frac{2 y z B}{r^{4}}(\dot{A} B-A \dot{B}), \\
H_{3}^{03}=\frac{2 B}{r^{2}}\left(\frac{z^{2}}{r^{2}}(\dot{A} B-A \dot{B})-(\dot{A} B+A \dot{B})\right) .
\end{gathered}
$$

Using the components (3.8) we obtain the components of energy and momentum densities in the form

$$
\begin{aligned}
& \theta_{0}^{0}=\frac{1}{8 \pi A r^{4}}\left(A^{2} r^{2}-B^{2}\right) \\
& \theta_{1}^{0}=\frac{B x}{4 \pi r^{4}}(\dot{A} B+A \dot{B}) \\
& \theta_{2}^{0}=\frac{B y}{4 \pi r^{4}}(\dot{A} B+A \dot{B}) \\
& \theta_{3}^{0}=\frac{B z}{4 \pi r^{4}}(\dot{A} B+A \dot{B})
\end{aligned}
$$

Using equations (3.8) in equation (3.7), the energy and momentum distributions are the following

$$
\begin{gathered}
E_{\text {Ein }}=P_{0}=\frac{1}{2 A r}\left(A^{2} r^{2}+B^{2}\right), \\
P_{1}=P_{2}=P_{3}=0 .
\end{gathered}
$$

We notice that if the signature of the space-time under study is changed from +2 to -2 , we find that the values of energy and momentum densities as well as the energy distribution are changed from positive to negative.

\section{The Energy-Momentum Complex of Bergmann- Thomson}

The Bergmann-Thomson energy-momentum complex [7] is given by

$$
\mathbf{B}^{i k}=\frac{1}{16 \pi}\left[g^{i l} \mathcal{B}_{l}^{k m}\right]_{, m},
$$


where

$$
\mathcal{B}_{l}^{k m}=\frac{g_{l n}}{\sqrt{-g}}\left[-g\left(g^{k n} g^{m p}-g^{m n} g^{k p}\right)\right]_{, p} .
$$

$B^{00}$ and $B^{0 \alpha}$ are the energy and momentum density components.

The energy and momentum are given by

$$
P^{i}=\iiint B^{i 0} d x^{1} d x^{2} d x^{3} .
$$

Using the Gauss theorem we have

$$
P^{i}=\frac{1}{16 \pi} \iint \mathcal{B}^{i 0 \alpha} n_{\alpha} d S
$$

In order to calculate the energy and momentum distributions for the space-time under consideration, using Bergmann-Thomson energy-momentum complex, we require the following non-vanishing components of $\mathcal{B}_{l}^{k m}$

$$
\begin{gathered}
\mathcal{B}_{0}^{01}=\frac{2 x}{A r^{4}}\left(A^{2} r^{2}+B^{2}\right), \\
\mathcal{B}_{0}^{02}=\frac{2 y}{A r^{4}}\left(A^{2} r^{2}+B^{2}\right), \\
\mathcal{B}_{0}^{03}=\frac{2 z}{A r^{4}}\left(A^{2} r^{2}+B^{2}\right), \\
\mathcal{B}_{1}^{01}=\frac{2 B}{r^{2}}\left(\frac{x^{2}}{r^{2}}(\dot{A} B-A \dot{B})-(\dot{A} B+A \dot{B})\right), \\
\mathcal{B}_{2}^{01}=\mathcal{B}_{1}^{02}=\frac{2 x y B}{r^{4}}(\dot{A} B-A \dot{B}), \\
\mathcal{B}_{3}^{01}=\mathcal{B}_{1}^{03}=\frac{2 x z B}{r^{4}}(\dot{A} B-A \dot{B}), \\
\mathcal{B}_{2}^{02}=\frac{2 B}{r^{2}}\left(\frac{y^{2}}{r^{2}}(\dot{A} B-A \dot{B})-(\dot{A} B+A \dot{B})\right), \\
\mathcal{B}_{3}^{02}=\mathcal{B}_{2}^{03}=\frac{2 y z B}{r^{4}}(\dot{A} B-A \dot{B}), \\
\mathcal{B}_{3}^{03}=\frac{2 B}{r^{2}}\left(\frac{z^{2}}{r^{2}}(\dot{A} B-A \dot{B})-(\dot{A} B+A \dot{B})\right) .
\end{gathered}
$$

Using the components (4.4) in (4.1), the components of energy and momentum densities are as follows

$$
\begin{gathered}
\mathbf{B}^{00}=\frac{1}{8 \pi A r^{4}}\left(A^{2} r^{2}-B^{2}\right), \\
\mathbf{B}^{01}=-\frac{x}{8 \pi r^{4}}\left(\dot{A} r^{2}+\frac{B}{A^{2}}(2 A \dot{B}-\dot{A} B),\right. \\
\mathbf{B}^{02}=-\frac{y}{8 \pi r^{4}}\left(\dot{A} r^{2}+\frac{B}{A^{2}}(2 A \dot{B}-\dot{A} B),\right. \\
\mathbf{B}^{03}=-\frac{z}{8 \pi r^{4}}\left(\dot{A} r^{2}+\frac{B}{A^{2}}(2 A \dot{B}-\dot{A} B) .\right.
\end{gathered}
$$

Using equations (4.4) in equation (4.3), we obtain the energy and momentum distributions in the following form

$$
E_{B e r g}=P^{0}=\frac{1}{2 A r}\left(A^{2} r^{2}+B^{2}\right),
$$




$$
P_{1}=P_{2}=P_{3}=0 .
$$

The above energy density and energy distribution are agreement with that obtained before, using Einstein's energy-momentum complex. In the case of Bergmann's energy-momentum complex, we notice that a signature switch do not affect about everything including energy distribution. Consequently, the Einstein and Bergmann-Thomson prescription do not give the same results when the signature of the space-time under study is -2 .

\section{Landau-Lifshitz's Energy-momentum Complex}

Landau-Lifshitz's energy-momentum complex [5] is given by

$$
L^{i j}=\frac{1}{16 \pi} S_{, k l}^{i k j l},
$$

where

$$
S^{i k j l}=-g\left(g^{i j} g^{k l}-g^{i l} g^{k j}\right) .
$$

$L^{i j}$ is symmetric in its indices, $L^{00}$ is the energy density and $L^{0 \alpha}$ are the momentum (energy current) density components. $S^{i k j l}$ has the symmetries of the Riemann curvature tensor.

The energy and momentum are given by

$$
P^{i}=\iiint L^{i 0} d x^{1} d x^{2} d x^{3} .
$$

Using the Gauss theorem we have

$$
P^{i}=\frac{1}{16 \pi} \iint S^{i 0 \alpha} n_{\alpha} d S
$$

The required non-vanishing components of $S^{i k j l}$ are

$$
\begin{array}{cc}
S^{0101}=B^{2}\left[\frac{x^{2}}{r^{6}}\left(A^{2} r^{2}-B^{2}\right)-\frac{A^{2}}{r^{2}}\right], \\
S^{0102} & =\frac{x y}{r^{6}} B^{2}\left(A^{2} r^{2}-B^{2}\right), \\
S^{0103} & =\frac{x z}{r^{6}} B^{2}\left(A^{2} r^{2}-B^{2}\right), \\
S^{0202}=B^{2}\left[\frac{y^{2}}{r^{6}}\left(A^{2} r^{2}-B^{2}\right)-\frac{A^{2}}{r^{2}}\right], & =\frac{y z}{r^{6}} B^{2}\left(A^{2} r^{2}-B^{2}\right), \\
S^{0203} & B^{0303}=B^{2}\left[\frac{z^{2}}{r^{6}}\left(A^{2} r^{2}-B^{2}\right)-\frac{A^{2}}{r^{2}}\right] .
\end{array}
$$


Using these components in equation (5.1), we obtained the energy and momentum densities are

$$
\begin{array}{cc}
L^{00} & =-\frac{B^{2}}{8 \pi r^{6}}\left[A^{2} r^{2}+3 B^{2}\right], \\
L^{10} & =-\frac{x B}{4 \pi}\left[A(A \dot{B}+\dot{A} B) r^{2}+2 B^{2} \dot{B}\right], \\
L^{20} & =-\frac{y B}{4 \pi r^{6}}\left[A(A \dot{B}+\dot{A} B) r^{2}+2 B^{2} \dot{B}\right], \\
L^{30} & =-\frac{z B}{4 \pi r^{6}}\left[A(A \dot{B}+\dot{A} B) r^{2}+2 B^{2} \dot{B}\right] .
\end{array}
$$

Using equations (5.1) in equation (5.4), we obtain the energy and momentum distributions

$$
\begin{aligned}
E_{L L} & =\frac{B^{2}}{2 r^{3}}\left(A^{2} r^{2}+B^{2}\right) . \\
P_{L L}^{1} & =P_{L L}^{2}=P_{L L}^{3}=0 .
\end{aligned}
$$

The above results do not agree with the results obtained before, using Einstein and Bergmann-Thomson energy-momentum complexes. A signature switch does not affect about everything including energy distribution.

\section{Papapetrou's Energy-momentum Complex}

The symmetric energy-momentum complex of Papapetrou [3] is given by

$$
\Omega^{i j}=\frac{1}{16 \pi} \Upsilon_{, k l}^{i j k l}
$$

where

$$
\Upsilon^{i j k l}=\sqrt{-g}\left(g^{i j} \eta^{k l}-g^{i k} \eta^{j l}+g^{k l} \eta^{i j}-g^{j l} \eta^{i k}\right),
$$

and $\eta^{i k}$ is the Minkowski metric with signature +2 .

$\Omega^{00}$ and $\Omega^{\alpha 0}$ are the energy and momentum density components.

The energy and momentum, using the Papapetrou prescription are given by

$$
P^{i}=\iiint \Omega^{i 0} d x^{1} d x^{2} d x^{3}
$$

Using the Gauss theorem we obtain

$$
P^{i}=\frac{1}{16 \pi} \iint \Upsilon_{, l}^{i 0 \alpha l} n_{\alpha} d S .
$$


The non-vanishing components of $\Upsilon^{i j k l}$ are as follows

$$
\begin{array}{cc}
\Upsilon^{0011} & =\frac{1}{A}\left[\frac{x^{2}}{r^{4}}\left(A^{2} r^{2}-B^{2}\right)-A^{2}\right]-\frac{A B^{2}}{r^{2}}, \\
\Upsilon^{0012} & =\frac{x y}{A r^{4}}\left(A^{2} r^{2}-B^{2}\right), \\
\Upsilon^{0013} & =\frac{x z}{A r^{4}}\left(A^{2} r^{2}-B^{2}\right), \\
\Upsilon^{0022} & =\frac{1}{A}\left[\frac{y^{2}}{r^{4}}\left(A^{2} r^{2}-B^{2}\right)-A^{2}\right]-\frac{A B^{2}}{r^{2}}, \\
\Upsilon^{0023} & =\frac{y z}{A r^{4}}\left(A^{2} r^{2}-B^{2}\right), \\
\Upsilon^{0033}=\frac{1}{A}\left[\frac{z^{2}}{r^{4}}\left(A^{2} r^{2}-B^{2}\right)-A^{2}\right]-\frac{A B^{2}}{r^{2}} .
\end{array}
$$

Using these components in (6.1), we get the following energy and momentum density components

$$
\begin{array}{cc}
\Omega^{00} & =\frac{A}{8 \pi r^{4}}\left[r^{2}-B^{2}\right] \\
\Omega^{10} & =-\frac{x}{8 \pi r^{4}}\left[r^{2} \dot{A}+B(\dot{A} B+2 A \dot{B})\right], \\
\Omega^{20} & =-\frac{y}{8 \pi r^{4}}\left[r^{2} \dot{A}+B(\dot{A} B+2 A \dot{B})\right], \\
\Omega^{30} & =-\frac{z}{8 \pi r^{4}}\left[r^{2} \dot{A}+B(\dot{A} B+2 A \dot{B})\right] .
\end{array}
$$

Using equations (6.5) in equation (6.4), we obtain the energy and momentum distributions

$$
\begin{aligned}
& E_{P}=\frac{A}{2 r}\left(r^{2}+B^{2}\right) \\
& P^{1}=P^{2}=P^{3}=0
\end{aligned}
$$

The above results do not agree with the results obtained before, using Einstein, Bergmann-Thomson and Landau and Lifshitz energy-momentum complexes. A signature switch should affect about everything including energy distribution.

\section{Discussion}

We investigated the energy and momentum (due to matter plus fields including gravity) distribution of the Kantowski and Sachs space-time using the Einstein, Bergmann-Thomson, Landau-Lifshitz and Papapetrou energymomentum complexes. We found that the quantities of energy and momentum densities as well as energy distribution are well-defined and wellbehaved. For the space-time under consideration, we found that the energymomentum complexes of Einstein and Bergmann-Thomson give the same results, while Landau-Lifshitz and Papapetrou do not give the same results and not agree with the aforementioned complexes. We have shown that a 
signature switch affects about every thing (by changing the sign of the values of energy and momentum densities as well as energy distribution) including energy distribution. These changes occur in the case of Einstein and Papapetrou prescriptions but not in Bergmann-Thomson and Landau-Lifshitz prescriptions.

\section{References}

[1] A. Einstein, Sitzungsber. Preuss. Akad. Wiss. Berlin (Math. Phys.) 778 (1915).

[2] R. C. Tolman, "Relativity, Thermodynamics and Cosmology, (Oxford University Press, Oxford), p. 227 (1934).

[3] A. Papapetrou, Proc. R. Ir. Acad. A52, 11 (1948).

[4] C. Møller, Ann. Phys. (NY) 4, 347 (1958).

[5] L. D. Landau and E. M. Lifshitz, "The Classical Theory of Fields", (Addison-Wesley Press, Reading, MA) p. 317 (1951).

[6] S. Weinberg, "Gravitation and Cosmology: Principles and Applications of General Theory of Relativity" ( Wiley, New York) 165 (1972).

[7] P. G. Bergmann and R. Thompson, Phys. Rev. 89, 400 (1953).

[8] K. S. Virbhadra, Phys. Rev D41, 1086 (1990).; K. S. Virbhadra, Phys. Rev. D42, 1066 (1990).; K. S. Virbhadra, Phys. Rev. D42, 2919 (1990).; K. S. Virbhadra, Pramana-J. Phys. 45, 215 (1995); N. Rosen, K.S. Virbhadra, Gen. Rel. Grav. 25, 429 (1993).

[9] J. M. Aguirregabiria, A. Chamorro and K.S. Virbhadra, Gen. Relativ. Gravit. 28, 1393 (1996).

[10] K. S. Virbhadra, Phys. Rev. D60, 104041 (1999).

[11] S. S. Xulu, Astrophys.Space Sci. 283, 23 (2003).

[12] R.M. Gad, Astrophys. Space Sci. 293, 453 (2004); R.M. Gad, Astrophys. Space Sci. 295, 459 (2005); R.M. Gad, Mod. Phys. Lett. A19, 1847 (2004); R.M. Gad, Gen. Relativ. Gravit., 38, 417 (2006); R. M. Gad, Astrophys.Space Sci. 302, 141 (2006); E. C. Vagenas, Int. J. Mod. 
Phys. A18 5781 (2003); E. C. Vagenas, Int. J. Mod. Phys. A18 5949 (2003); E. C. Vagenas, Mod. Phys. lett. A 19, 213 (2004); E. C. Vagenas, Int.J.Mod.Phys.D 14,573 (2005); E. C. Vagenas, Mod. Phys. Lett. A 21, 1947 (2006); I.-C. Yang, C.-T. Yeh, R.-R. Hsu and C.-R. Lee, Int. J. Mod. Phys. D 6, 349 (1997); I. Radinschi, Acta Phys. Slov. 49, 789 (1999); I. Radinschi, Mod. Phys. Lett. A 15, 803 (2000); I. Radinschi, Acta Phys. Slov. 50, 609 (2000); I. Radinschi, FizikaB 9, 43 (2000); I. Radinschi, Nuovo Cim. B 115, 501 (2000); I. Radinschi, Mod. Phys. Lett. A 15, 2171 (2000); I. Radinschi, Mod. Phys. Lett. A 16, 673, (2001); I. Radinschi, Chin. J. Phys. 39, 393 (2001); I.-C. Yang and I. Radinschi, Chin. J. Phys. 41, 326 (2003); I.-C. Yang and I. Radinschi, Mod. Phys. Lett. A 17, 1159 (2002); I. Radinschi, Chin. J. Phys. 39, 393 (2001); I. C. Yang and I. Radinschi, Chin. J. Phys. 42, 40 (2004); I. Radinschi and I. C. Yang, FizikaB 14, 3 (2005); I. Radinschi, Rom. J. Phys. 50, 57 (2005); I. Radinski and Th. Grammenos, Int. J. Mod. Phys. A 21, 2853 (2006); I.-C. Yang, Chin. J. Phys. 38, 1040 (2000); O. Aydogdu and M. Salti, Prog. Theor. Phys. 115, 63 (2006); O. Aydogdu, M. Salti and M. Korunur, Acta Phys. Slov. 55, 537 (2005); M. Salti, Acta Phys. Slov. 55, 563 (2005); M. Salti, Nuovo Cim. 120B, 53 (2005); O. Aydogdu and M. Salti, Astrophys. Space Sci. 299, 227 (2005); M. Salti, Mod. Phys. Lett. A 20, 2175 (2005); M. Salti and A. Havare, Int. J. Mod. Phys. A 20, 2169 (2005); A. Havare, M. Korunur and M. Salti, Astrophys. Space Sci. 301, 43 (2006); O. Patashnick, Int. J. Mod. Phys. D 14, 1607 (2005); T. Grammenos, Mod. Phys. Lett. A 20, 1741 (2005).

[13] C. W. Misner, K. S. Thorne and J. A. Wheeler, "Gravitation" (Freeman W. H and Co., NY) p. 603 (1973).

[14] F. I. Cooperstock and R. S. Sarracino, J. Phys. A 11877 (1978).

[15] H. Bondi, Proc. R. Soc. London A 427, 249 (1990).

[16] F. I. Cooperstock, Found. Phys. 22, 1011 (1992); in Topics in Quantum Gravity and Beyond: Pepers in Honor of L, Witten eds. F. Mansouri and J. J. Scanio (World Scientific, Singapore, (1993)) 201; In Relativistic Astrophysics and Cosmology, eds. Buitrago et al. (World Scientific, Singapore, (1997))61; Annals Phys. 282, 115 (2000).

[17] S. S. Xulu, Mod. Phys. Lett. A15, 1511; Astrophys. and Space Science 283, 23 (2003). 
[18] R. M. Gad, Astrophys. space Sci., 295, 451 (2005).

[19] R. Kantowski and R. K. Sachs, J. Math. Phys. 7, 443 (1966).

[20] C. B. Collins, J. Math. Phys. 18, 2116 (1977).

[21] A. S. Kompaneets and Chernov A. S., Sov. Phys. JETP 20, 1303 (1965). 